\title{
Review of Recommendations for Gynecology Practice during COVID-19 Pandemic
}

\author{
Amrutha Kakollu ${ }^{1}$ Anupama Hari² \\ ${ }^{1}$ Department of Obstetrics and Gynecology, KIMS Hospital, \\ Secunderabad, India \\ 2Department of Obstetrics and Gynecology, Malla Reddy College, \\ Telangana, India \\ Ind J Car Dis Wom:2020;5:230-232
}

\begin{abstract}
Address for correspondence Amrutha Kakollu, MS, FMAS, Department of Obstetrics and Gynecology, KIMS Hospital, Secunderabad, India (e-mail: amrutha9jaideep@gmail.com).
\end{abstract}

\author{
Abstract \\ keywords \\ - abnormal uterine \\ bleeding \\ - covid19 \\ - gynecology
}

The impact of COVID-19 on healthcare is enormous. It is essential that we prioritize treating patients and reserving our resources for better care of patients affected with COVID-19. The well-being of the healthcare professionals is of utmost importance as well. Treating gynecological patients during this time is challenging as individualizing treatment without compromising safety and minimizing risk is of utmost importance. In this article, we have aimed to write a consensus to guide the treatment of gynecological patient, based on the limited evidence available as of today.

\section{Introduction}

The COVID-19 pandemic has represented a major impact to health systems and the society in general all around the world, and it has forced many changes that will affect our practice for an amount of time that is still hard to predict.

With obstetrics comprising a major component of priority healthcare, most gynecology patients who are planned for elective surgery can be offered medical treatment, with exceptions in the form of patients with menorrhagia, causing severe anemia, postmenopausal bleeding or malignancy.

Routine screening of patients for COVID-19 with clinical, laboratory and radiological investigations is essential before consulting any gynecologic patient in person.

In this article, we have mainly reviewed the guidelines provided by American College of Obstetricians and Gynecologists (ACOG), Royal College of Obstetrics and Gynecology (RCOG), as the guidelines specific to gynecology practice are limited.

\section{Triaging Gynecological Patients}

ACOG recommend prioritizing gynecologic patients based on local availability of resources and patient population. ${ }^{1}$

Patients with conditions like severe gynecologic infections, suspected ectopic pregnancy, and major postoperative complications requiring admission should be advised to consult in person.
Women who require contraception advice, follow-up of asymptomatic ovarian cyst, and routine postoperative checkup can avail of these services via teleconsultation. ${ }^{1}$

\section{Routine Gynecologic Checkup}

Patients requesting for a routine gynecologic check up can be deferred until the burden of the pandemic subsides.

\section{Recommended Outpatient Practices}

The recommendations to modify routine practice are as follows ${ }^{1}$ :

- Universal mask policy for healthcare professionals, patients, and visitors.

- Restricting the number of attendants.

- Social distancing to be practiced in elevators, waiting rooms, and during examination clinic spaces.

- Hand sanitizing options for patients and staff members to be used before entering the clinic.

- Offering teleconsultation wherever possible.

- Planning patient visits by prior appointment to prevent crowding of waiting area.

- Minimize visits by preplanning the investigations the patient might need and addressing them in the same visit.
(C)2020 Women in Cardiology and Related Sciences
License terms

()(1) $\Theta \circledast$ 


\section{Benign Gynecological Conditions}

\section{Heavy Menstrual Bleeding (HMB)}

In the treatment of HMB, RCOG recommends initial management of the patient by teleconsultation to assess the severity of symptoms. As the risk of malignancy is less in these women, patients can be reassured of the same and provided medical treatment at home after excluding contraindications and severe anemia/risk factors for malignancy.

If the patient has severe acute bleeding resulting in severe anemia, then she can be treated with tranexamic acid or acute high-dose progesterone or gonadotropin-releasing hormone $(\mathrm{GnRH})$ analogues. Correction of anemia can be done with oral/parenteral iron or a blood transfusion depending on severity. ${ }^{2}$

When the patient cannot be planned for surgery immediately, GnRH analogues can be given to arrest bleeding, especially when there are associated fibroids. Alternative treatment in women with acute episode of heavy bleeding is to give high dose of oral progestogens (e.g., norethisterone up to $15 \mathrm{mg} /$ day; medroxyprogesterone acetate $15-30 \mathrm{mg}$ /day) to be administered to arrest acute episodes of bleeding (single 10-14 day course).

\section{Postcoital Bleeding}

Women with postcoital bleeding can be reassured that the risk of cervical malignancy is very less if she has had a recent normal cervical smear.

However, if the woman does not have a recent cervical smear or her previous smear is abnormal, she would need to attend the hospital for a cervical examination. ${ }^{2}$

\section{Postmenopausal Bleeding}

Women with postmenopausal bleeding (PMB) can be managed through telecommunication to confirm the symptom and assess the urgency of in person consultation. The patient can be assessed for symptoms of COVID-19 and advised accordingly.

Women who have suspected or confirmed COVID-19should be advised to access appropriate care and consult further after they are not infectious and retested. However, the risk of delayed treatment should be considered, especially in high risk of malignancy.

Evaluation of postmenopausal women should comprise a speculum examination as a normal cervix in women with a negative cervical smear excludes cervical cancer.

Transvaginal ultrasound (TVS) with measurement of the endometrial thickness (ET) should be performed, and women with an ET of $<4 \mathrm{~mm}$ on TVS can be reassured, as the risk of endometrial malignancy is very minimal. If the ET is $>4 \mathrm{~mm}$, endometrial sampling should be advised, based on the resources available. If the histopathology is inadequate or benign, patient can be reassured.

There is no increased risk of transmission of SARSCoV-2 to healthcare professionals during a hysteroscopy, blind endometrial biopsy, and polypectomy. Best practice is to minimize contamination from blood, urine, genital tract fluids, and feces during any genital tract procedure.
Simultaneous insertion of levonorgestrel intrauterine device can be considered during endometrial biopsy or hysteroscopy in selected cases. ${ }^{2}$

\section{Cancer Cervix Screening by Colposcopy}

During the COVID-19 crisis, only women with a recent cervical smear suggesting high grade, moderate neoplasia in endocervical cells or possible glandular neoplasia/invasive disease should be seen for colposcopy.

RCOG consensus states that in asymptomatic women, gloves, apron and an appropriate mask be worn for colposcopy consultation and examination.

A minimum number of staff should be present during procedures. A serviced smoke evacuator must be used for laser loop excision procedures. Laser ablation and excision should not be used due to vaporization and aerosol generation.

In women with suspected or confirmed COVID-19 infection, colposcopy assessment should be postponed until symptoms resolve or the woman has tested negative. Precautions should be taken by instructing women not to cough during the procedure to facilitate bringing the cervix into view.

If women have symptoms suggestive of cervical cancer and are symptomatic of COVID-19, patient should be seen at the end of OPD and preferably in a separate COVID designated area. The colposcopy team should wear full personal protective equipment (PPE) during consultation and examination. $^{3}$

\section{Elective Gynecological Surgeries}

Elective procedures should be postponed and patients offered alternative conservative measures when possible. Patients with abnormal uterine bleeding and menorrhagia, resulting in severe anemia, can be taken up for surgery after appropriate screening for COVID-19 infection. All operating room staff should be following universal precautions with adequate PPE to minimize exposure and risk of infection. ${ }^{1,2}$

\section{Contraception}

Contraception care needs to be simplified in accordance with the current pandemic demanding enormous resources. Teleconsulting can be done in women who can self-administer medication and who need refill of their prescription.

For patients requesting emergency contraception, longacting reversible contraceptive methods (LARC) should be offered like intrauterine contraceptive device; implants and permanent contraception can be offered when possible. If there is inability to immediately offer the above, the patient can be given oral contraceptives for self-administration during this period of delay. Women with LARC scheduled for removal can be postponed and counselled for extended usage and benefits. ${ }^{1,4}$ 


\section{Gynecologic Oncology}

The Society of Gynecologic Oncology recommends continuing evaluation and treatment of patients who are newly diagnosed with malignancy, prioritizing higher grade pathology, patients with recurrent cancer and symptoms that require immediate treatment. ${ }^{5}$

Among patients with ovarian cancer, neoadjuvant therapy can be considered to delay surgery. The dose of chemotherapy needs to be carefully adjusted to minimize neutropenia.

Women diagnosed with endometrial cancer, who are at a higher risk of perioperative morbidity, can be offered alternative treatment options like hormonal therapy for intraepithelial neoplasia/low-grade malignancy.

The treatment of patients diagnosed with preinvasive cervical malignancy can be deferred. Women with operable disease can be taken up for surgery, as planned, and patients with inoperable disease should be treated with therapeutic chemoradiation as required

\section{Conclusion}

To conclude, the current COVID-19 pandemic has significantly changed our day-to-day practice. In this scenario, safety of the healthcare staff is as important as the patient's safety. Therefore trying to implement the above mentioned guidelines in out patient can reduce the risk of exposure to covid 19 for the treating physician, hospital staff and other patients attending the clinic. Proper screening, both clinical and by laboratory, and radiological investigations are essential in triaging patients with high risk of active COVID-19 disease. The take-home message is that whenever applicable, medical or nonsurgical treatment should be preferred, and surgery should be postponed while balancing the risk of disease progression with treatment delay.

\section{Conflicts of Interest}

None declared.

\section{References}

1 American College of Obstetricians and Gynecologists. COVID-19 FAQs for Obstetricians-Gynecology. Available at: https://www.acog.org/clinical-information/physician-faqs/ covid19-faqs-for-ob-gyns-gynecology. Accessed August 24, 2020

2 Guidance for the Management of Abnormal Uterine Bleeding in the Evolving Coronavirus (COVID-19) Pandemic. Available at: https://www.rcog.org.uk/globalassets/ documents/guidelines/2020-05-21-joint-rcog-bsge-bgcsguidance-for-management-of-abnormal-uterine-bleedingaub-in-the- evolving-coronavirus-covid-19-pandemic-updated-final-180520.pdf. Accessed August 24, 2020

3 BSCCP and RCOG Colposcopy Guidance During COVID-19 Pandemic https://www.rcog.org.uk/ globalassets/documents/ guidelines/2020-04-17-colposcopy-guidanc-during-covid-19pandemic.pdf. Accessed August 24, 2020

4 COVID-19 Resources and Information for Sexual and Reproductive Healthcare Professionals. Available at: https:// www.fsrh.org/fsrh-and-covid-19-resources-and-informationfor-srh/. Accessed August 24, 2020

5 Gynecologic Oncology Considerations During the COVID-19 Pandemic. Available at: https://www.sgo.org/ resources/ gyn-onc-considerations-during-covid-19/. Accessed August 24, 2020 